\title{
Non-typhoidal Salmonella abscess in a patient with a large thymoma: a rare presentation and review of the literature
}

Irene J. Lo ${ }^{1}$, Wilbert S. Aronow², Irlna I. Tantchou ${ }^{1}$, Rocco Lafaro ${ }^{3}$, Abraham Marav $^{1}$, Arif Muslim¹, Suryanarayana Pothula ${ }^{1}$

${ }^{1}$ Department of Surgery, Division of General Surgery, Westchester Medical Center/ New York Medical College, Valhalla, NY, USA

${ }^{2}$ Department of Medicine, Division of Cardiology, Westchester Medical Center/ New York Medical College, Valhalla, NY, USA

${ }^{3}$ Department of Cardiovascular Surgery, Westchester Medical Center/New York Medical College, Valhalla, NY, USA

Submitted: 12 April 2013

Accepted: 12 April 2013

Arch Med Sci 2015; 11, 2: 453-457

DOI: 10.5114/aoms.2015.50979

Copyright (C) 2015 Termedia \& Banach

Salmonella infection commonly occurs in the gastrointestinal tract secondary to fecal-oral transmission of the bacterium. Extraintestinal Salmonella is typically seen in the biliary system and is very rarely observed in the thoracic cavity. Salmonella infection in the thymus is an even more unusual presentation of extraintestinal Salmonella, with only one previous case reported in the literature. Thymic abscesses are already a rare clinical entity and have been reported to result from infection with Staphylococcus aureus. We report a case of a 59-year-old man with a non-typhoidal Salmonella infection of a large symptomatic thymoma that was treated with surgical drainage and antibiotic therapy.

A 59-year-old Pakistani man was admitted to our hospital in January, 2013 with a large, symptomatic anterior mediastinal mass. During the year prior to admission at our institution, the patient had become progressively more short of breath and had significantly decreased exercise tolerance. The patient was also experiencing intermittent fevers and leukocytosis. The patient was evaluated in Pakistan by a cardiologist, who performed an echocardiogram and a stress test. The echocardiogram revealed a mediastinal and right pleural base mass.

Additional radiological imaging was subsequently obtained to further evaluate this mass. A chest X-ray was obtained which confirmed a large mass in the right pleural space. Computed tomography (CT) scan of the chest also demonstrated a large inhomogeneous mass with almost near total compression of the right atrium and total compression of the lung with significant compression atelectasis. A biopsy of the mass was performed which indicated a diagnosis of thymoma.

The patient traveled to the United States for further work-up and management. A CT scan of the chest obtained in Pakistan revealed a persistent right pleural based mass with compression of the right atrium and right lung (Figure 1). The pathology slides were reviewed by two separate institutions, and the diagnosis was confirmed to be thymoma. After discussion with the patient and his family regarding treatment options, the risks of surgery, and the benefits of surgery, the patient elected to undergo surgical resection of the mass. During his
Corresponding author: Wilbert S. Aronow MD, FACC, FAHA

Cardiology Division New York Medical College Macy Pavilion, Room 138 Valhalla, NY 10595

Phone: (914) 4935311

Fax: (914) 2356274

E-mail:wsaronow@aol.com 


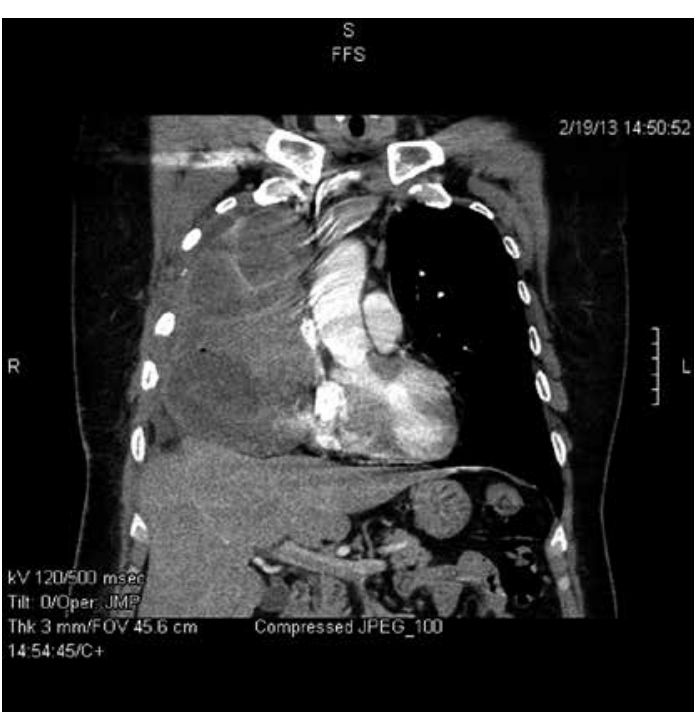

Figure 1. Initial CT scan obtained in Pakistan

pre-operative evaluation, the patient was noted to be febrile to $103^{\circ}$ with significant leukocytosis of 30,000 on his laboratory results. The patient was admitted to the thoracic surgery service for further work-up.

The patient underwent a chest X-ray which continued to show a large right pleural mass (Figure 2). A repeat transthoracic echocardiogram was performed, which showed normal ventricular function, no vegetations, and continued compression of the right atrium by the tumor mass. A Duplex ultrasound of the patient's lower extremities was performed, which showed no deep venous thromboses. Blood and urine cultures were also obtained and were negative. The hematology/ oncology service was consulted, and a bone marrow biopsy and peripheral smear were performed, which were both negative. The Infectious Disease service was also consulted for management of antibiotic therapy, and the patient was started on intravenous antibiotics. At this point, given our negative work-up, the patient's intermittent fevers and leukocytosis were thought to be secondary to compression atelectasis of the patient's right lung as well as massive tumor necrosis.

The patient was taken to the operating room on hospital day 2 for surgical resection of this mass. In the operating room, the patient was endotracheally intubated with a double-lumen endotracheal tube and transesophageal echocardiography was performed. The patient was placed in the left lateral decubitus position, and an extended right posterolateral thoracotomy incision was made. The patient's right pleural cavity was subsequently explored. An enormous mass was demonstrated, which involved all three lobes of the right lung. The mass was opened, and a large amount of purulent fluid and necrotic tumor mass

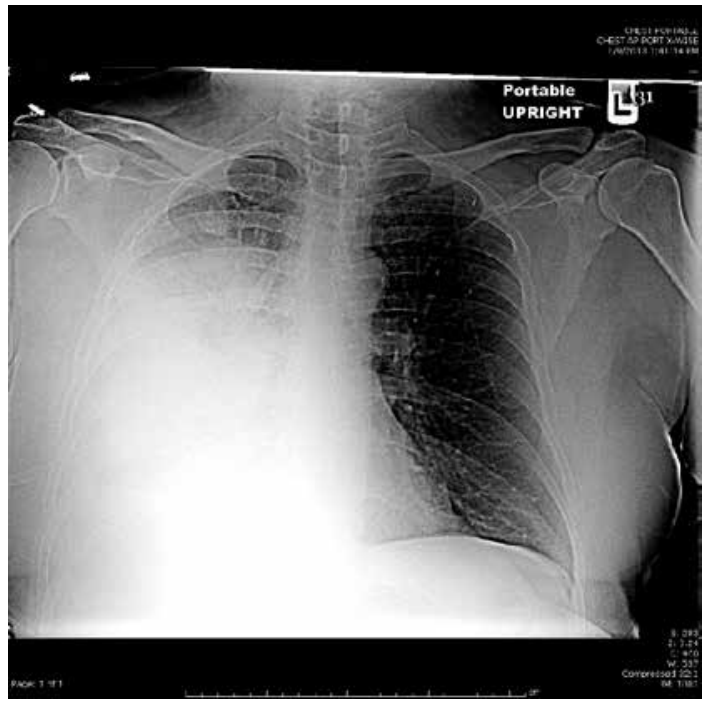

Figure 2. Chest X-ray obtained preoperatively demonstrates hypoinflation of the right lung

was obtained. The fluid was sent for culture and the mass for frozen section.

The frozen section was reported to be suspicious for lymphoma, with definitive diagnosis deferred to permanent section after review by three separate senior pathologists. After discussion with the patient's oncologist, the decision was made to defer resection of the mass, which might require a pneumonectomy, until a definitive diagnosis could be obtained. Chest tubes were placed for drainage and the patient's wound was closed in layers. The patient was then transferred in intubated condition to the cardiothoracic intensive care unit.

On the first postoperative day, the patient became septic, requiring vasopressor support and aggressive fluid resuscitation. Over the next couple of postoperative days, the patient was weaned off vasopressors. The patient was also weaned off the ventilator and extubated on postoperative day 2 . The patient was subsequently transferred to the medical/surgical unit. Chest tubes remained in place until drainage was minimal. Daily chest X-rays were obtained during the course of his hospitalization, and noticeable improvement was noted in the patient's right lung (Figure 3). Final pathology was consistent with multiple tumor fragments composed predominantly of T cells, interspersed with scattered round to oval neoplastic epithelial cells with vesicular nuclei - consistent with type B1 (lymphocytic rich) thymoma. The final microbiology report showed Salmonella species, non-typhoidal. Intravenous antibiotic coverage was therefore changed to a better targeted therapy in the form of cefepime.

The patient was transitioned to oral antibiotics and was discharged home on post-operative day 10 with continued antibiotic therapy and 


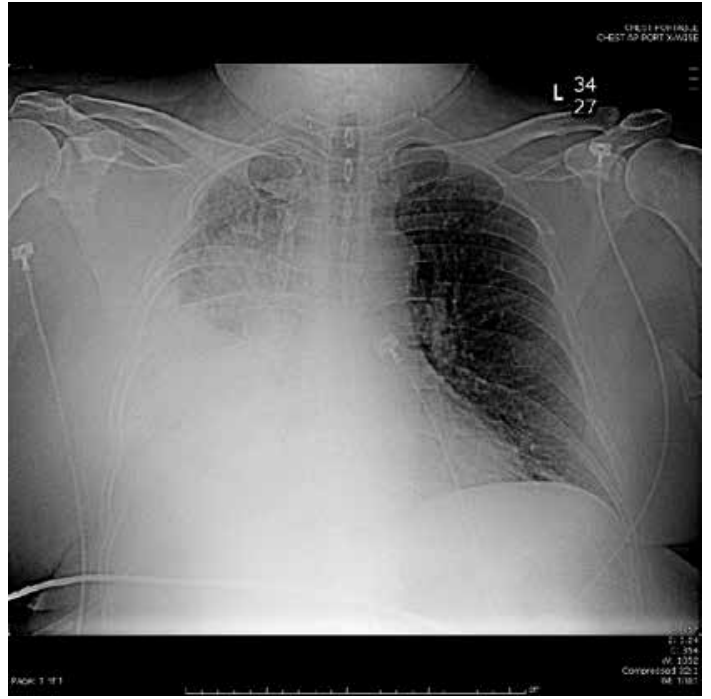

Figure 3. Chest X-ray obtained postoperatively already shows better aeration of the right lung after thymic abscess was drained in initial operation

a follow-up plan that included having him return for a complete resection of the mass or conservative management if the mass on repeat $\mathrm{CT}$ had resolved or increased with antibiotic therapy only. Four weeks following the first procedure, the patient obtained a repeat CT (Figure 4) that showed the presence of the mass without any significant change in the size of the mass, and the patient was doing well but continued to have some effort-induced shortness of breath.

After discussion with the patient and his family, the decision was then made to undergo surgical resection of this mass. He was readmitted to our institution on March 14, 2013. He underwent a right thoracotomy with complete resection of the tumor mass from the right pleural space with partial right lower lobectomy on March 16, 2013. On an echocardiogram that was performed intraoperatively, a large mass compressing the right atrium was noted (Figure 5). Once the specimen was completely resected (Figure 6), another

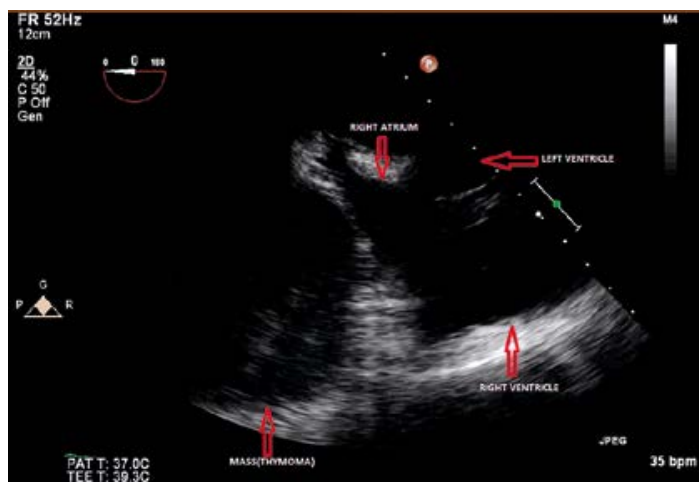

Figure 5. Echocardiogram in the long axis view demonstrating the right atrium being compressed by the mass

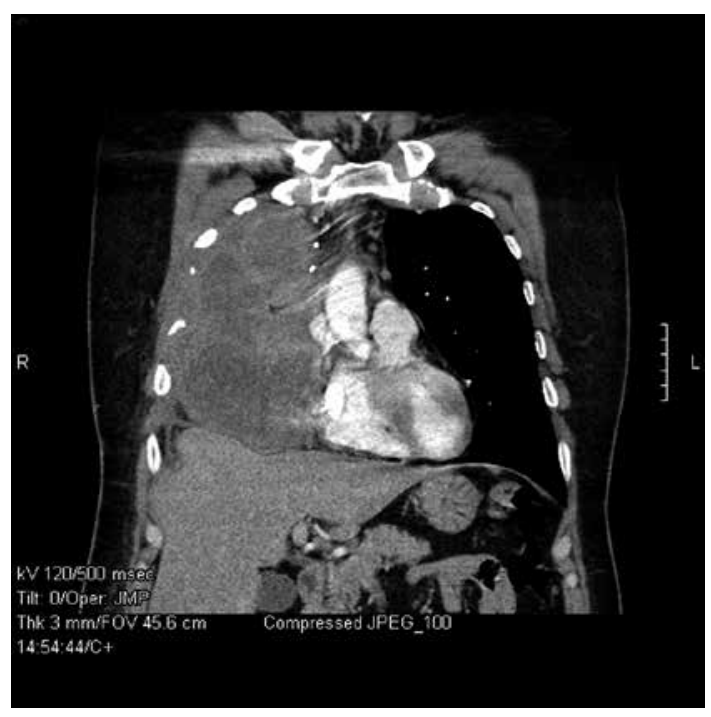

Figure 4. The CT scan demonstrating the large mediastinal mass occupying almost the entire right thoracic cavity

echocardiogram was again done, which showed a marked improvement in the compression of the right atrium by the mass that had occupied almost all of the thoracic cavity on the right side (Figure 7). He tolerated the procedure well without immediate complications.

His postoperative course was uneventful. He remained in the intensive care unit for 1 day and was transferred to a surgical floor on postoperative day 2. His shortness of breath resolved. A significant improvement on chest $X$-ray was also noted (Figure 8). The chest tube that had been placed intraoperatively was discontinued on postoperative day 2 , and he was sent home on postoperative day 5 to complete 1 week of antibiotic therapy.

Thymomas are the most common neoplasm found in the anterior compartment of the mediastinum. Thymomas originate from thymic epithelial cells. Generally, patients with thymomas are asymptomatic, but many patients may have symp-

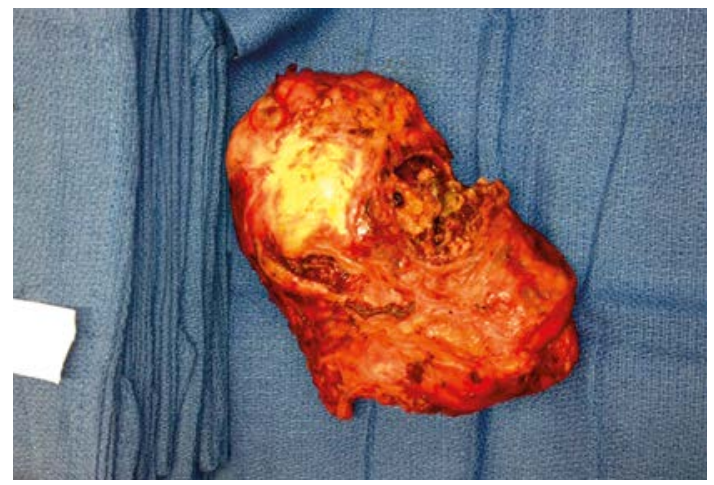

Figure 6. Specimen obtained after complete resection 


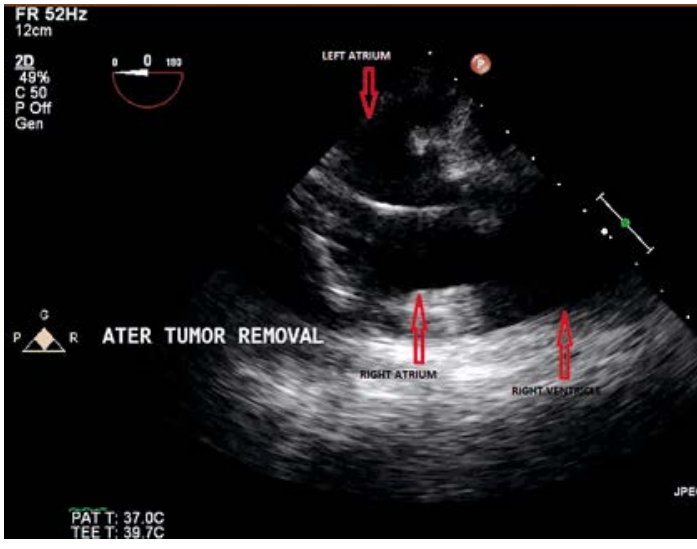

Figure 7. The right atrium cavity appears larger after the mass is resected

toms associated with a large thymoma compressing nearby structures or with myasthenia gravis. Patients with thymoma are generally treated with surgical resection, and the prognosis is excellent for patients with early disease and complete resection. Given the location of our patient's mass, our leading diagnosis was thymoma, which was confirmed by the results of a core needle biopsy performed at an outside institution. As a result, given the patient's presumptive diagnosis and the patient's symptomatology, our decision was to proceed with surgical resection.

T-cell lymphomas are also found in the anterior mediastinum and must also be considered in the differential diagnosis of patients with anterior mediastinal neoplasms. During the frozen section analysis of a specimen from our patient's tumor, the pathologists could not determine a definitive diagnosis, with lymphoma and thymoma among the possible diagnoses. However, because lymphoma was a consideration and lymphomas are generally treated non-surgically with chemotherapy and radiation, our decision was to defer resection of the mass until a definitive diagnosis could be obtained. If the tumor was lymphoma, treatment would be deferred to the oncologists, and if the tumor turned out to be thymoma, then we would proceed with resection at a later date.

Our patient did not simply have a thymoma. In addition to a large anterior mediastinal mass, our patient also had an abscess, secondary to Salmonella infection. This posed a serious therapeutic dilemma. Salmonella is generally a disease entity limited to the gastrointestinal tract, with extraintestinal disease commonly found in the biliary tract. Thoracic Salmonella is rare, and thymic Salmonella is even rarer. Salmonella infection also generally occurs in patients who are immunocompromised. His thymoma may have led to an immunocompromised state which predisposed him to a Salmonella infection, whose cause remains unknown.

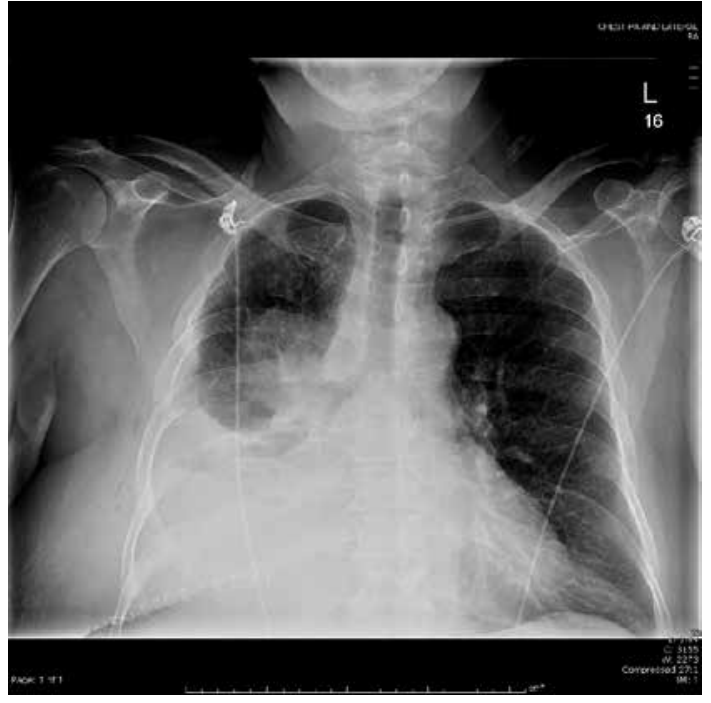

Figure 8. Chest X-ray obtained after second operation that demonstrates improved aeration of the right lung

There has only been one previous case report describing Salmonella bacteremia associated with a thymic abscess within a thymoma [1]. In this report, Carter et al. [1] suggested a multimodal approach. Using CT of the chest, they were able to obtain an accurate definition of the mass, with respect to malignant potential, and also develop a surgical plan, using the delineated anatomy. They also recommended percutaneous drainage of the mass and abscess to provide for stabilization of the patient prior to surgical resection. Carter et al. [1] pursued surgical resection of their patient's mass once her sepsis resolved. Specifically, they performed an en bloc resection of the thymus, decortication, and copious irrigation through a median sternotomy, which they felt would adequately treat the infection process as well as any potential neoplasms.

There have also been previous case reports regarding a similar clinical entity - mediastinitis involving Salmonella. These studies have recommended drainage, either surgical or percutaneous, or surgical exploration of the mediastinum in cases of mediastinitis involving Salmonella [2-5]. One particular report specifically recommended surgical exploration as preferable because this type of drainage and exploration would not only be diagnostic, but also therapeutic at the same time. Antibiotic therapy should also be tailored to culture results.

However, the timeline to surgical resection in the setting of infection still remains unclear. The patient described by Carter et al. [1] underwent a wedge resection of the medial aspect of the lower lobe. In our patient, however, in order to obtain en bloc resection of the mass, a right pneumonectomy was possible. In the setting of an infection 
that had not been adequately treated, this posed a significant risk to the patient as a potential complication would be blowout of the patient's bronchial stump. Also, our patient had already undergone an extended right thoracotomy in his first surgical procedure, and the question arose as to what type of exposure would be needed in his definitive operation to gain access to the mass.

Although cases of thymoma and Salmonella abscesses are rare entities, adequate management of these cases must include en bloc resection of the mass, decortication, and copious irrigation. Through this approach, the patient's mass is resected in its entirety, and the infectious process is treated as well. Our patient's treatment plan involved initial drainage of the abscess and mass, followed by definitive surgical resection once the patient's infection had been adequately treated so as to minimize any potential postoperative complications. As more cases are encountered and managed medically and surgically, more optimal treatment protocols and timelines may be established. However, it is also important that approaches to treatment are also tailored to the patient in question.

\section{Conflict of interest}

The authors declare no conflict of interest.

\section{References}

1. Carter YM, Shagrun L, Klein H, et al. Salmonella infection in an anterior mediastinal mass. J Thorac Cardiovasc Surg 2005; 129: 228-30.

2. Marsh MD, Amorosa JK, Abassi M, et al. Salmonella mediastinitis - a rare disease. Comput Radiol 1984; 9: 375-7.

3. Tilly H, Gardembas M, Barry M, et al. Salmonella infection in hairy cell leukemia: report of a case. Am J Hematol 1985; 19: 423-5.

4. Snider JM, Hannah H, McNabney WK, Pemberton LB. Salmonella mediastinal abscess. Ann Thorac Surg 1993; 56: 556-68.

5. Rubinstien E, Slavin J. Thymic abscess with bacteremia and manubriosternal pyarthrosis in a geriatric patient. Chest 1993; 103: 962-4. 